Chatsworth, CA) and cloned using the pCR-Script kit (Stratagene). A minimum of 8 clones from each culture were sequenced on an automated sequencer (LI-COR, Lincoln, NE). Sequences were aligned manually with other marine cyanobacterial sequences available in the Ribosomal Database Project ${ }^{28}$ using the Genetic Data Environment ${ }^{29}$. The sequence for MIT9303 obtained previously from a PCR product ${ }^{17}$ is contained within the sequence we report here. A total of 1,094 unambiguously aligned and determined nucleotides were used in the analyses. Phylogenetic analyses used PAUP* (version 4.0d47, provided by D. Swofford). For both distance and maximum likelihood analyses the model of nucleotide substitution used was the Hasegawa Kishino Yana 1985 model. Nucleotide frequencies and the transition transversion ratio were estimated from the data.

Received 11 November 1997; accepted 26 March 1998.

1. Chisholm, S. W. et al. A novel free-living prochlorophyte abundant in the oceanic euphotic zone Nature 334, 340-343 (1988).

2. Chisholm, S. W. et al. Prochlorococcus marinus nov. gen. nov. sp.: an oxyphototrophic marine prokaryote containing divinyl chlorophyll $a$ and $b$. Arch. Microbiol. 157, 297-300 (1992).

3. Olson, R. J., Chisholm, S. W., Zettler, E. R., Altabet, M. A. \& Dusenberry, J. A. Spatial and temporal distributions of prochlorophyte picoplankton in the North Atlantic Ocean. Deep Sea Res. 37, 1033 1051 (1990).

4. Campbell, L. \& Vaulot, D. Photosynthetic picoplankton community structure in the subtropica North Pacific Ocean near Hawaii (station ALOHA). Deep Sea Res. 40, 2043-2060 (1993).

5. Goericke, R. \& Repeta, D. J. Chlorophylls $a$ and $b$ and divinyl chlorophylls $a$ and $b$ in the open subtropical North Atlantic Ocean. Mar. Ecol. Prog. Ser. 101, 307-313 (1993).

6. Moore, L. R., Goericke, R. \& Chisholm, S. W. Comparative physiology of Synechococcus and Prochlorococcus: influence of light and temperature on growth, pigments, fluorescence and absorptive properties. Mar. Ecol. Prog. Ser. 116, 259-275 (1995).

Giovannoni, S. J., Britschgi, T. B., Moyer, C. L. \& Field, K. G. Genetic diversity in Sargasso Sea bacterioplankton. Nature 345, 60-63 (1990).

8. Fuhrman, J. A., McCallum, K. \& Davis, A. A. Phylogenetic diversity of subsurface marine microbia communities from the Atlantic and Pacific Oceans. Appl. Environ. Microbiol. 59, 1294-1302 (1993)

9. Palenik, B. Cyanobacterial community structure as seen from RNA polymerase gene sequence analysis. Appl. Environ. Microbiol. 60, 3212-3219 (1994).

10. DeLong, E. F., Wu, K. Y., Prèzelin, B. B. \& Jovine, R. V. M. High abundance of Archaea in Antarctic marine picoplankton. Nature 371, 695-697 (1994).

11. Ward, D., Weller, M. R. \& Bateson, M. M. 16S rRNA sequences reveal numerous uncultured microorganisms in a natural community. Nature 345, 63-65 (1990).

12. Amann, R., Snaidr, J., Wagner, M., Ludwig, W. \& Schleifer, K.-H. In situ visualization of high genetic diversity in a natural microbial community. J. Bacteriol. 178, 3496-3500 (1996).

13. Veldhuis, M. J. W. \& Kraay, G. W. Cell abundance and fluorescence of picoplankton in relation to growth irradiance and nitrogen availability in the Red Sea. Neth. J. Sea. Res. 31, 135-145 (1993).

14. Partensky, F., Blanchot, J., Lantoine, F., Neveaux, J. \& Marie, D. Vertical structure of picophytoplankton at different trophic sites of the subtropical northeastern Atlantic Ocean. Deep Sea Res. 43, $1191-1213$ (1996)

15. Toledo, G. \& Palenik, B. Synechococcus diversity in the California Current as seen by RNA polymerase (rpoC1) gene sequences of isolated strains. Appl. Environ. Microbiol. 63, 4298-4303 (1997).

16. Shimada, A., Maruyama, T. \& Miyachi, S. Vertical distributions and photosynthetic action spectra of two oceanic picophytoplankters, Prochlorococcus marinus and Synechococcus sp. Mar. Biol. 127, 15-23 (1996).

17. Urbach, E., Scanlan, D. J., Distel, D. L., Waterbury, J. B. \& Chisholm, S. W. Rapid diversification of marine picophytoplankton with dissimilar light-harvesting structures inferred from sequences of Prochlorococcus and Synechococcus (Cyanobacteria). J. Mol. Evol. 46, 188-201 (1998).

18. Partensky, F., Hoepffner, N., Li, W. K. W., Ulloa, O. \& Vaulot, D. Photoacclimation of Prochlorococcus sp. (Prochlorophyta) strains isolated from the north Atlantic and the Mediterranean Sea. Plant Physiol. 101, 285-296 (1993).

19. Scanlan, D. J., Hess, W. R., Partensky, F., Newman, J. \& Vaulot, D. High degree of genetic variation in Prochlorococcus (Prochlorophyta) revealed by RFLP analysis. Eur. J. Phycol. 31, 1-9 (1996).

20. Moore, L. R. \& Chisholm, S. W. Photophysiology of the marine cyanobacterium Prochlorococcus: ecotypic differences among cultured isolates. Limnol. Oceanogr. (submitted).

21. Waterbury, J. B., Watson, S. W., Valois, F. W. \& Franks, D. G. Biological and ecological characterization of the marine unicellular cyanobacterium Synechococcus. Can. Bull. Fish. Aquat. Sci. 214, 71-120 (1986).

22. Olson, R. J., Chisholm, S. W., Zettler, E. R. \& Armbrust, E. V. Analysis of Synechococcus pigment types in the sea using single and dual beam flow cytometry. Deep Sea Res. 35, 425-440 (1988).

23. Gallagher, J. C. Population genetics of Skeletonema costatum (Bacillariophyceae) in Narrangansett Bay. J. Phycol. 16, 464-474 (1980).

24. Gallagher, J. C. Physiological variation and electrophoretic banding patterns of genetically differen seasonal populations of Skeletonema costatum (Bacillariophyceae). J. Phycol. 18, 148-162 (1982).

25. Platt, T., Gallegos, C. L. \& Harrison, W. G. Photoinhibition of photosynthesis in natural assemblages of marine phytoplankton. J. Mar. Res. 38, 687-701 (1980).

26. Jeffrey, S. W. \& Humphrey, G. F. New spectrophotometric equations for determining chlorophyll $a, b$ $c_{1}$ and $c_{2}$ in higher plants, algae, and natural phytoplankton. Biochem. Physiol. Pflanz. 168, 191-194 (1975).

27. Ausubel, F. M. et al. Short Protocols in Molecular Biology (John Wiley \& Sons, New York, 1992).

28. Maidak, B. L. et al. The RDP (Ribosomal Database Project). Nucleic Acids Res. 25, 109-111 (1997).

29. Smith, S. W., Overbeek, R., Woese, C. R. \& Gilbert, W. The genetic data environment: an expandable GUI for multiple sequence analysis. CABIOS 10, 671-675 (1994).

Acknowledgements. We thank M. Sogin for access to an automated DNA sequencer and helpfu discussion; A. Shimada for the use of unpublished sequences of Prochlorococcus GP2 and SB; R. Olson and E. Zettler for cell sorting at sea; H. Hsu for technical assistance; and C. Cavanaugh, H. Sosik and S. Sathyendranath for helpful consultation. This work was supported by the US National Science Foundation and the US National Aeronautics and Space Administration.

Correspondence and requests for materials should be addressed to S.W.C. (e-mail: chisholm@mit.edu). Sequences have been deposited in GenBank under accession numbers AF053396, MIT9302; AF053397, MIT9303; AF053398, MIT9312; and AF053399, MIT9313.

\section{Conscious and unconscious emotional learning in the human amygdala}

\section{J. S. Morris ${ }^{\star}$, A. Öhman $\dagger$ \& R. J. Dolan ${ }^{\star}$}

* Wellcome Department of Cognitive Neurology, Queen Square, London WC1N 3BG, UK

$\dagger$ Department of Clinical Neuroscience, Section of Psychiatry and Psychology, Karolinska Hospital, S-17176 Stockholm, Sweden

$\ddagger$ Royal Free and University College Hospitals School of Medicine,

Rowland Hill Street, London NW3 2DF, UK

If subjects are shown an angry face as a target visual stimulus for less than forty milliseconds and are then immediately shown an expressionless mask, these subjects report seeing the mask but not the target. However, an aversively conditioned masked target can elicit an emotional response from subjects without being consciously perceived $^{1,2}$. Here we study the mechanism of this unconsciously mediated emotional learning. We measured neural activity in volunteer subjects who were presented with two angry faces, one of which, through previous classical conditioning, was associated with a burst of white noise. In half of the trials, the subjects' awareness of the angry faces was prevented by backward masking with a neutral face. A significant neural response was elicited in the right, but not left, amygdala to masked presentations of the conditioned angry face. Unmasked presentations of the same face produced enhanced neural activity

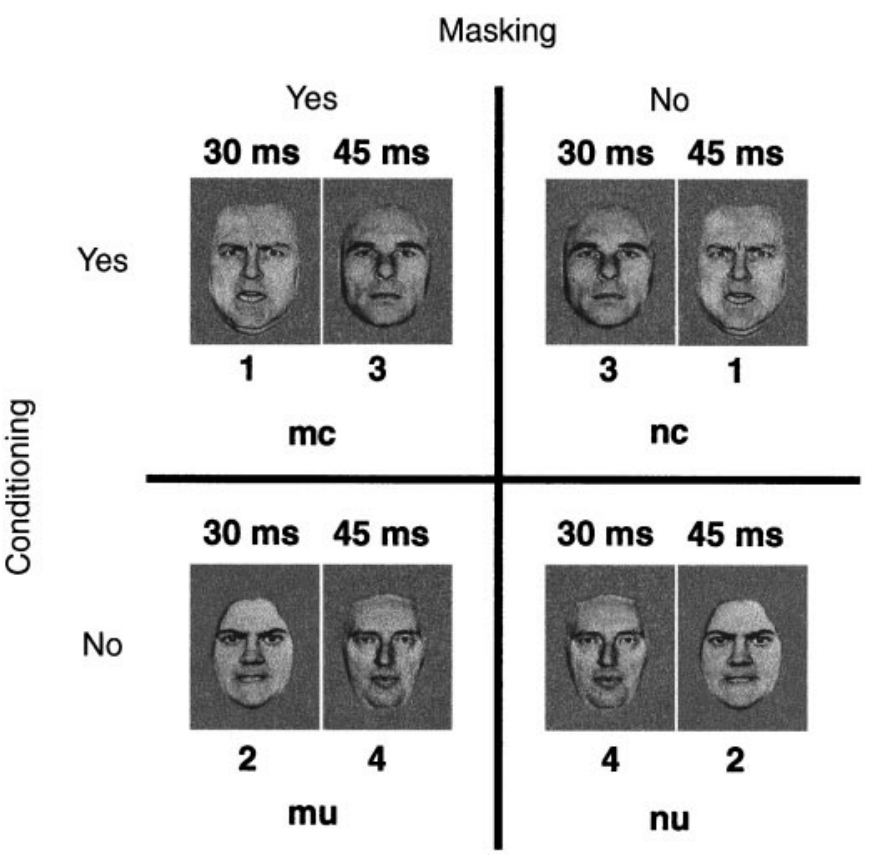

Figure 1 Stimulus parameters and experimental design. In the scanning window, pairs of target and masking faces were shown in four separate conditions, determined by the combination of masking and conditioning of the angry face. Mc, masked conditioned (the CS+ angry face was the target and the neutral face was the mask); nc, non-masked conditioned (a neutral face was the target and the CS+ face was the mask); mu, masked unconditioned (the CS-face was the target and the neutral face the mask); nu, non-masked unconditioned (the neutral face was the target and the CS- face the mask). Face 1, angry face paired with noise $(\mathrm{CS}+$ ); face 2, angry face not paired with noise (CS-); faces 3 and 4, neutral faces. In all conditions, the target face was displayed for $30 \mathrm{~ms}$ and immediately followed by the mask for $45 \mathrm{~ms}$. 
in the left, but not right, amygdala. Our results indicate that, first, the human amygdala can discriminate between stimuli solely on the basis of their acquired behavioural significance, and second, this response is lateralized according to the subjects' level of awareness of the stimuli.

Studies of animals ${ }^{3-6}$ and brain-damaged patients $s^{7-10}$ indicate a crucial role for the amygdala in emotional learning. Other data show that the amygdala can respond differentially to emotional stimuli that subjects are unable explicitly to recall ${ }^{11,12}$. However, direct evidence for the involvement of the amygdala in the unconscious mediation of learned emotional responses ${ }^{1,2}$ has been lacking. In this experiment we used a factorial design to measure directly how neural responses associated with emotional learning are modulated by subjects' conscious awareness (Fig. 1). We showed healthy volunteer subjects pictures of two angry faces, one of which was paired $(\mathrm{CS}+)$, and the other unpaired (CS-), with an unconditioned stimulus (UCS), namely, a $100-\mathrm{dB}$ burst of white noise. Following this conditioning, the faces were presented sequentially, either masked or unmasked, and without the UCS, while neural activity was measured by positron emission tomography (PET). Throughout the experiment, subjects were required to indicate, by pressing a button, any occurrence of either angry face. Skin conductance responses (SCRs) were measured during both conditioning and scanning phases.

The responses of subjects revealed an inability to detect the masked angry faces: $0 \%$ of the masked angry faces were detected, whereas $100 \%$ of the unmasked faces were detected. On subsequent a

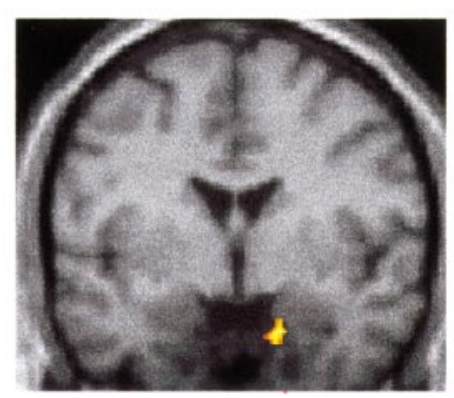

Coronal

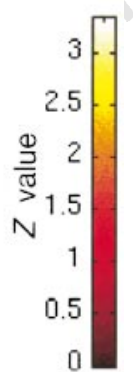

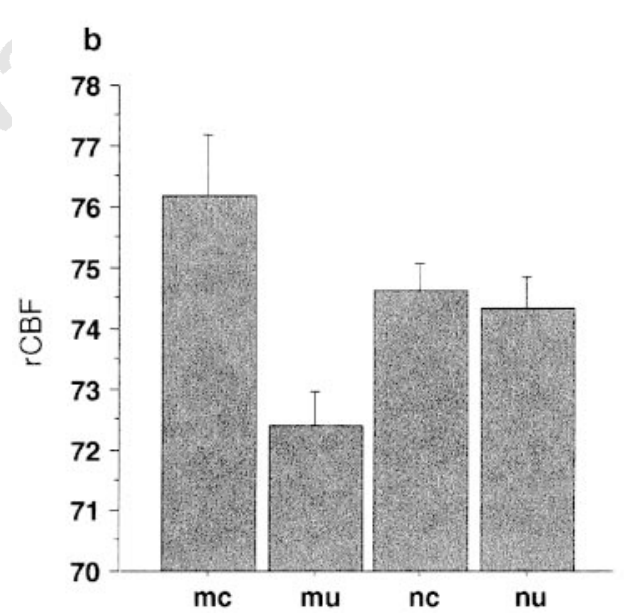

Figure 2 Response to the presentation of masked faces. a, An SPM showing activation in the region of the right amygdala in the contrast of masked CS+ and CS- angry faces. An uncorrected threshold of $P=0.01$ was used to display the contrasts. The SPM is displayed on a coronal slice $(y=-2 \mathrm{~mm})$ of a canonical MRI image. b, A graphical display of the mean regional cerebral blood flow (rCBF) in the four conditions at the maximal voxel of activation in the right amygdala, $x=18, y=-2, z=-28$. Bars represent two standard errors. See Fig. 1 legend for abbreviations. debriefing, two subjects reported awareness of 'flickering' occurring on masked presentations, but did not report any perception of the angry faces. The remaining eight subjects denied any awareness of the masking procedure. Mean SCRs were significantly greater for $\mathrm{CS}+$ than for CS- faces $(P<0.01)$, both for masked (CS+ mean $=0.605 \mu \mathrm{S}$, s.e.m. $=0.06 ;$ CS - mean $=0.473$, s.e.m. $=$ $0.09 \mu \mathrm{S})$ and unmasked $(\mathrm{CS}+$ mean $=0.748 \mu \mathrm{S}$, s.e.m. $=0.044$; CS - mean $=0.426 \mu$ S, s.e.m. $=0.084)$ presentations.

Bilateral responses in the amygdala were detected in scans of brain responses to all CS+ versus all CS- faces (masked and unmasked) (Table 1). To determine whether this response was present when subjects could not report the masked faces, we contrasted activity only in the masked CS+ and CS- scans. There was significant response $(P<0.05$, corrected $)$ in the region of the right amygdala to the presentation of masked CS+ faces (Fig. 2 and Table 1). This right-sided focus of activation was located in a medial and inferior part of the amygdaloid complex. In electrophysiological recordings of monkey brains, a similar region of amygdala was aversive cues ${ }^{13}$. When contrasting responses to unmasked CS+ and CS- faces, we saw a significant response $(P<0.05$, corrected $)$ in the left amygdala (Fig. 3 and Table 1). This left-sided activation by reportable CS+ faces was located in a more superior and posterior region of the amygdala than the right-sided activation by masked $\mathrm{CS}+$ stimuli. Even with liberal thresholding, there was no evidence of responses of left amygdala to masked CS+ faces or of right amygdala to unmasked CS+ faces. Direct comparison of activity reported to contain units that are selective for faces and visually

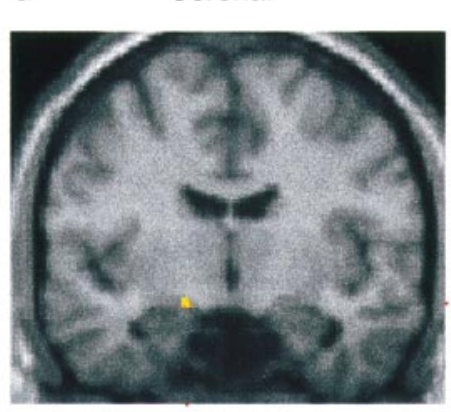

Coronal

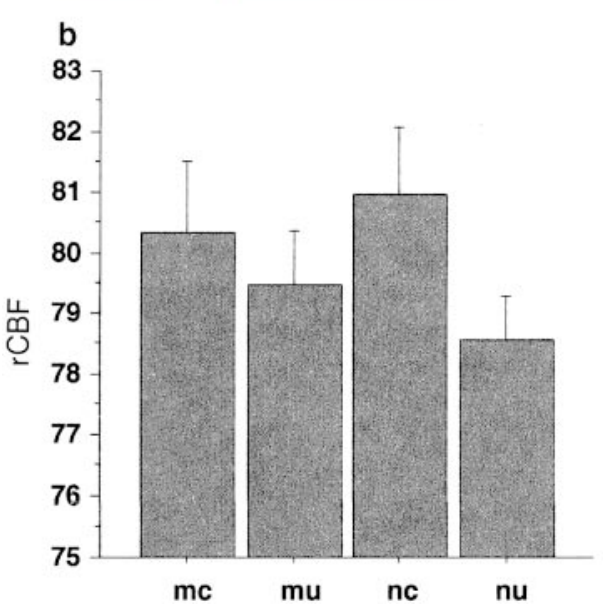

Figure 3 Response to the presentation of unmasked faces. a, A statistical parametric map (SPM) showing activation of the left amygdala in the contrast of unmasked CS+ and CS- angry faces. An uncorrected threshold of $P=0.01$ was used to display the contrasts. The SPM is displayed on a coronal slice $(y=-8 \mathrm{~mm})$ of a canonical MRI image. $\mathbf{b}$, A graphical display of the mean rCBF in the four conditions at the maximal voxel of activation in the left amygdala, $x=-18, y=-8, z=-14$. Bars represent two standard errors. See Fig. 1 legend for abbreviations. 


\begin{tabular}{|c|c|c|c|c|}
\hline & $\begin{array}{c}\text { Coordinates } \\
\quad(x, y, z)\end{array}$ & Voxels* & $Z$ score & $\begin{array}{c}P \text { value } \\
\text { (corrected) }\end{array}$ \\
\hline $\begin{array}{l}\text { All CS+ versus all } \\
\text { Left amygdala } \\
\text { Right amygdala }\end{array}$ & $\begin{array}{c}-16,-10,-14 \\
18,-2,-28\end{array}$ & $\begin{array}{r}6 \\
21\end{array}$ & $\begin{array}{l}2.86 \\
2.67\end{array}$ & $\begin{array}{l}0.02 \\
0.04\end{array}$ \\
\hline $\begin{array}{l}\text { Masked CS+ versu } \\
\text { Right amygdala }\end{array}$ & $\begin{array}{l}\text { asked CS- } \\
18,-2,-28\end{array}$ & 44 & 3.42 & 0.01 \\
\hline $\begin{array}{l}\text { Unmasked CS+ ve } \\
\text { Left amygdala }\end{array}$ & $\begin{array}{l}\text { unmasked CS- } \\
-16,-8,-14\end{array}$ & 6 & 2.92 & 0.02 \\
\hline
\end{tabular}

* Coordinates of the maximally activated voxel and the number of significant voxels are shown here.

between the two amygdalae revealed significant $(P<0.05)$ masking-related hemispheric effects.

We also formally tested the interaction between conditioning and masking, implicit in the above data, by comparing, for example, the responses to masked conditioned minus masked unconditioned faces with non-masked conditioned minus non-masked unconditioned faces. The results indicate that the response of the right amygdala to CS+ faces was significantly enhanced by masking (maximal voxel $x=18, y=-2, z=-28, Z=2.30, P<0.05$ ), whereas the response of the left amygdala was enhanced by unmasking (maximal voxel $x=-14, y=-8, z=-14, Z=1.65$, $P<0.05)$. As the different face stimuli were equally represented in conditions across subjects, and as no noise UCSs were played during scanning, the CS+ and CS- conditions involved identical physical stimuli and the same explicit task. The only difference, therefore, between the CS+ and CS- conditions was the subjects' previous experience of the temporal association between $\mathrm{CS}+$ faces and aversive noise.

Our results are evidence that neural activity in the human amygdala mediates the learning of associations between behaviourally significant stimuli. The results concur with studies of fear conditioning in animals $\mathrm{s}^{3-6}$ and of human lesions ${ }^{7-10}$ that also indicate that the amygdala is involved in emotional learning. The results are also consistent with neuroimaging data that show that amygdala activity during viewing of emotional stimuli correlates significantly with subsequent recall of the same stimuli ${ }^{14}$. A new aspect of our findings is that a response of the amygdala occurred to masked conditioned faces that were not consciously perceived. Although differential amygdala activity during passive viewing of masked fearful and happy faces has been reported ${ }^{12}$, the subjects' awareness of the stimuli was not measured at the time of presentation, thus limiting interpretation of the data in terms of unconscious processing. In our study, however, we directly assessed subjects' awareness during scanning by an explicit target-detection task. Also, here the differential response of the amygdala was related not to differences in emotional expression, but to categorically identical stimuli that differed solely in terms of their earlier associative history (that is, one was associated with the aversive UCS). In this respect, we provide the first evidence that the human amygdala can discriminate the acquired behavioural significance of stimuli without the need for conscious perception.

Another new finding is the lateralization of the amygdala response as a function of the level of awareness of target stimuli. The response of the right amygdala to masked CS+ faces concurs with previous findings of a right-hemisphere advantage for processing emotional facial expressions ${ }^{15-17}$. The absence of activity of the right amygdala in the unmasked condition, when subjects could report the occurrence of conditioned stimuli, indicates that processes related to conscious awareness, such as the engagement of language systems, may inhibit this neural response. This proposal is consistent with data from 'split-brain' patients which show that emotional visual stimuli presented to the right hemisphere produce greater autonomic responses when masked (and unreportable) than when unmasked ${ }^{18}$. The proposal also concurs with electrophysiological data from humans concerning single-unit responses to previously presented stimuli; greater responses of the amygdala are produced in response to target stimuli that subjects could not recall ${ }^{11}$. Also, psychophysical data show enhanced semantic priming with masked (unreportable) words; that is, there is attenuation of the priming effect by conscious awareness ${ }^{19}$. Our data may help to explain the failure of previous functional neuroimaging experiments using unmasked, reportable stimuli to show direct activation of the amygdala during conditioning paradigms ${ }^{20,21}$. Subjects' conscious processing of stimuli in these studies may have attenuated amygdala responses.

The response of the right amygdala was attenuated in the unmasked condition, whereas activity of the left amygdala was enhanced with awareness of the stimuli. Left-hemisphere involvement in the recognition of facial expressions has been shown in several studies of human lesions ${ }^{22,23}$. Previous functional imaging experiments have also reported left-lateralized responses in the amygdala that are related to the perception of facial expressions ${ }^{24,25}$. All these studies used stimuli that subjects could identify by explicit report, indicating that the response of the left amygdala to emotional stimuli may be enhanced by verbal or conscious processing. The absence of a significant response of the left amygdala in the masked condition, when conscious (verbal) processing was prevented, supports this view. Neuroimaging data showing activation of right $>$ left amygdala in response to masked fearful faces ${ }^{12}$, but activation of left $>$ right amygdala responses to the same stimuli without masking ${ }^{25}$, are consistent with this interpretation.

Finally, the lateralization of the amygdala response here is consistent with the behavioural responses of patients with cerebral commissurotomies. 'Split-brain' patients can verbally report stimuli presented to the isolated left hemisphere yet deny awareness of the same stimuli presented to the right hemisphere ${ }^{26}$. However, the isolated right hemisphere shows superior performance in tasks involving facial and emotional discrimination, when verbal processing is not critical $^{16,26}$. Greater heart-rate responses are provoked by masked emotional visual stimuli when presented to the right rather than left hemisphere of split-brain patients, but the same stimuli shown unmasked produce greater increases in heart rate after presentation to the isolated left hemisphere ${ }^{18}$. The lateralized amygdala activity here is consistent with these lesion data and indicates that the same segregation of conscious and unconscious processing that is observed in the split-brain may also be present in the intact brain.

\section{Methods}

Ten healthy, right-handed male subjects took part in the study. Subjects (mean age $32.7 \mathrm{yr}$ ) were recruited by advertisement. They all gave informed consent and the study was approved by the local hospital ethics committee and ARSAC (UK). Each subject was scanned 12 times for the distribution of $\mathrm{H}_{2}{ }^{15} \mathrm{O}$ using a Siemans/CPS ECAT EXACT HR + PET Scanner operated in high-sensitivity three-dimensional mode. Subjects received a total of $350 \mathrm{MBq}$ of $\mathrm{H}_{2}{ }^{15} \mathrm{O}$ over $20 \mathrm{~s}$ through a forearm cannula. Images were reconstructed into 63 planes, using a Hanning filter, resulting in $6.4-\mathrm{mm}$ transaxial and $5.7-\mathrm{mm}$ axial resolution (full width was half the maximum). Each scanning window was of duration $90 \mathrm{~s}$.

PET data were analysed using statistical parametric mapping (SPM96) software from the Wellcome Department of Cognitive Neurology, London ${ }^{27,28}$. After initial realignment, the PET scans were transformed into a standard stereotactic space ${ }^{28}$. Structural magnetic resonance images (MRIs) from each subject were co-registered into the same space. The scans were then smoothed using a Gaussian filter, set at $12 \mathrm{~mm}$ full width at half maximum. We adjusted the $\mathrm{rCBF}$ (regional cerebral blood flow) measurements to a global mean of $50 \mathrm{ml}$ per dl per min. A blocked (by subject) analysis of covariance model was fitted to the data at each voxel, with condition effects for each of the four masking variations, and global CBF as a confounding covariate. We assessed predetermined contrasts of the condition effects at each voxel using a $t$-statistic, 
giving a statistic image for each contrast. $P$ values for activations in the amygdala were corrected for the volume of brain analysed (specified as a sphere with radius $8 \mathrm{~mm})^{29}$. Anatomical localization for the group meancondition-specific activations are reported in standard space $e^{28}$. In all cases, the localization of the group mean activations was confirmed by registration with the subject's own MRIs.

In an initial conditioning phase immediately before scanning, subjects viewed a sequence of greyscale images of four faces taken from a standard set of pictures of facial affect ${ }^{30}$. Images of a single face were presented on a computer monitor screen for $75 \mathrm{~ms}$ at intervals of $15-25 \mathrm{~s}$ (mean $20 \mathrm{~s}$ ). Each of the four faces was shown six times in a pseudorandom order. Two of the faces had angry expressions (A1 and A2), the other two being neutral (N1 and N2). One of the angry faces (CS+) was always followed by a 1-s 100-dB burst of white noise. In half of the subjects A1 was the CS+ face; in the other half, A2 was used. None of the other faces was ever paired with the noise. Before each of the 12 scanning windows, which occurred at 8-min intervals, a shortened conditioning sequence was played consisting of three repetitions of the four faces. During the 90-s scanning window, which seamlessly followed the conditioning phase, 12 pairs of faces, consisting of a target and mask, were shown at 5-s intervals. The target face was presented for $30 \mathrm{~ms}$ and was immediately followed by the masking face for $45 \mathrm{~ms}$ (Fig. 1). These stimulus parameters remained constant throughout all scans and effectively prevented any reportable awareness of the target face (which might be a neutral face or an angry face).

There were four different conditions (Fig. 1), masked conditioned, nonmasked conditioned, masked unconditioned, and non-masked unconditioned. Throughout the experiment, subjects performed the same explicit task, which was to detect any occurrence, however fleeting, of the angry faces. Immediately before the first conditioning sequence, subjects were shown the two angry faces and were instructed, for each stimulus presentation, to press a response button with the index finger of the right hand if one the angry faces appeared, or another button with the middle finger of the right hand if they did not see either of the angry faces.

Throughout the acquisition and extinction phases, subjects' SCRs were monitored to index autonomic conditioning. SCRs were measured with Biodata galvanic skin response equipment using Ag/AgCl electrodes attached to the palmar surface of the middle phalanges of the index and middle fingers of the left hand. We took readings of skin conductance (in $\mu S$ ) every $500 \mathrm{~ms}$ and stored them digitally on computer. All SCRs were square-root-transformed to attain statistical normality. Using the SCR in the 4-s period before presentation as a baseline, the maximal SCR deflection in the period $0.5-4 \mathrm{~s}$ after a face was presented was assigned as the value for the SCR to that face. The mean SCRs for the CS+ and CS- angry faces were calculated for both the masked and the unmasked conditions, and the differences between the means were tested using a paired Student's $t$-test.

\section{Received 2 February; accepted 13 March 1998.}

1. Esteves, F., Dimberg, U. \& Öhman, A. Automatically elicited fear: conditioned skin conductance responses to masked facial expressions. Cogn. Emotion 9, 99-108 (1994).

2. Ohman, A. \& Soares, J. J. F. "Unconscious anxiety": phobic responses to masked stimuli. J. Abnorm Psychol. 103, 231-240 (1994).

3. Weiskrantz, L. Behavioural changes associated with ablation of the amygdaloid complex in monkeys. J. Comp. Physiol. Psychol. 49, 381-391 (1956).

4. Kapp, B. S., Pascoe, J. P. \& Bixler, M. A. in Neuropyschology of Memory (eds Butters, N. \& Squire, L. R.) 473-488 (Guilford, New York, 1984).

5. Davis, M. in The Amygdala: Neurobiological Aspects of Emotion, Memory and Mental Dysfunction (ed. Aggleton, J. P.) 255-306 (Wiley-Liss, New York, 1992).

6. LeDoux, J. E. The Emotional Brain (Simon and Schuster, New York, 1996)

7. Bechara, A. et al. Double dissociation of conditioning and declarative knowledge relative to the amygdala and hippocampus in humans. Science 269, 1115-1118 (1995).

8. LaBar, K. S., LeDoux, J. E., Spencer, D. D. \& Phelps, E. A. Impaired fear conditioning following unilateral temporal lobectomy. J. Neurosci. 15, 6846-6855 (1995).

9. Babinsky, R. et al. The possible contribution of the amygdala to memory. Behav. Neurol. 6, 167-170 (1993).

10. Adolphs, R., Cahill, L., Scull, R. \& Babinsky, R. Impaired declarative memory for emotional materia following bilateral amygdala damage in humans. Learning Memory 4, 291-300 (1997).

11. Fried, I., Macdonald, K. A. \& Wilson, C. L. Single neuron activity in hippocampus and amygdala during recognition of faces and objects. Neuron 18, 875-887 (1997).

12. Whalen, P. J. et al. Masked presentations of emotional facial expressions modulate amygdala activity without explicit knowledge. J. Neurosci. 18, 411-418 (1998).

13. Leonard, C. M., Rolls, E. T., Wilson, F. A. \& Baylis, G. C. Neurons int he amygdala of the monkey with responses selective for faces. Behav. Brain Res. 15, 159-176 (1985).

14. Cahill, L. et al. Amygdala activity at encoding correlated with long-term, free recall of emotional information. Proc. Natl Acad. Sci. USA 93, 8016-8021 (1996).

15. DeKosky, S. T., Heilman, K. M., Bowers, D. \& Valenstein, E. Recognition and discrimination of emotional faces and pictures. Brain Lang. 9, 206-214 (1980).
6. Gazzaniga, M. S. \& Smylie, C. S. Facial recognition and brain asymmetries: clues to underlying mechanisms. Ann. Neurol. 13, 536-540 (1983).

17. Gur, R. C., Skolnick, B. E. \& Gurr, R. E. Effects of emotional discrimination tasks on cerebral blood flow: regional activation and its relation to performance. Brain Cogn. 25, 271-286 (1994).

18. Ladavas, E., Cimatti, D., Del Pesce, M. \& Tuozzi, G. Emotional evaluation with and without conscious stimulus identification: evidence from a split-brain patient. Cogn. Emotion 7, 95-114 (1993).

19. Klinger, M. R. \& Greenwald, A. G. Unconscious priming of association judgements. J. Exp. Psych. Learn. Mem. Cogn. 21, 569-581 (1995).

20. Hugdahl, K. et al. Brain mechanisms in human classical conditioning: a PET blood flow study. Neuroreport 6, 1723-1728 (1995).

21. Morris, J. S., Friston, K. J. \& Dolan, R. J. Neural responses to salient visual stimuli. Proc. R. Soc. Lond. B 264, 769-775 (1997).

22. Young, A. W., Newcombe, F., de Haan E. H. F., Small, M. \& Hay, D. C. Face perception after brain injury. Selective impairments affecting identity and expression. Brain 116, 941-959 (1993).

23. Stone, V. S., Nisenson, L., Eliassen, J. C. \& Gazzaniga, M. S. Left hemisphere representations of emotional facial expressions. Neuropsychol. 34, 23-29 (1995).

24. Morris, J. S. et al. A differential neural response in the human amygdala to fearful and happy facial expressions. Nature 383, 812-815 (1996).

25. Breiter, H. C. et al. Response and habituation of the human amygdala during visual processing of facial expression. Neuron 17, 875-887 (1996).

26. Gazzaniga, M. S. in The Cognitive Neurosiciences (ed. Gazzaniga, M. S.) 1049-1061 (MIT Press, Cambridge, MA, 1995).

27. Friston, K. J. et al. Statistical parametric maps in functional imaging: a general linear approach. Hum. Brain Mapp. 2, 189-210 (1995)

28. Friston, K. J. et al. Spatial registration and normalization of images. Hum. Brain Mapp. 3, 165-189 (1995).

29. Worsley, K. J., Marrett, P., Neelin, A. C., Friston, K. J. \& Evans, A. C. A unified statistical approach for determining significant signals in images of cerebral activation. Hum. Brain Mapp. 4, 58-73 (1996). 30. Ekman, P. \& Friesen, W. V. Pictures of Facial Affect (Consulting Psychologists Press, Palo Alto, 1976).

\section{The human amygdala in social judgment}

\section{Ralph Adolphs ${ }^{\star}$, Daniel Tranel ${ }^{\star}$ \& Antonio R. Damasio ${ }^{\star} \dagger$}

* Department of Neurology, Division of Cognitive Neuroscience, University of Iowa College of Medicine, 200 Hawkins Drive, Iowa City, Iowa 52242, USA $\dagger$ The Salk Institute for Biological Studies, La Jolla, California 92037, USA

Studies in animals have implicated the amygdala in emotional ${ }^{1-3}$ and social ${ }^{4-6}$ behaviours, especially those related to fear and aggression. Although lesion ${ }^{7-10}$ and functional imaging ${ }^{11-13}$ studies in humans have demonstrated the amygdala's participation in recognizing emotional facial expressions, its role in human social behaviour has remained unclear. We report here our investigation into the hypothesis that the human amygdala is required for accurate social judgments of other individuals on the basis of their facial appearance. We asked three subjects with complete bilateral amygdala damage to judge faces of unfamiliar people with respect to two attributes important in real-life social encounters: approachability and trustworthiness. All three subjects judged unfamiliar individuals to be more approachable and more trustworthy than did control subjects. The impairment was most striking for faces to which normal subjects assign the most negative ratings: unapproachable and untrustworthy looking individuals. Additional investigations revealed that the impairment does not extend to judging verbal descriptions of people. The amygdala appears to be an important component of the neural systems that help retrieve socially relevant knowledge on the basis of facial appearance.

Data from three subjects with complete bilateral amygdala damage (subjects SM, JM and $\mathrm{RH}$ ) and seven with unilateral amygdala damage were compared to those from normal and from brain-damaged control subjects (see Table 1 and Methods). Ratings of approachability and of trustworthiness were analysed separately for the 50 faces to which normal controls assigned the most negative ratings, and for the 50 most positive faces. Subjects with bilateral amygdala damage rated the 50 most negative faces more positively than did either normal controls $(P<0.01)$ or brain-damaged controls $(P<0.05$; Mann-Whitney $U$-tests on subjects' mean ratings, Bonferroni corrected) (Fig. 1). Groups with unilateral amygdala lesions did not differ from controls on either rating. All subject groups gave similar ratings to the 50 most positive faces. 